\title{
La adherencia terapéutica en el paciente trasplantado renal
}

\author{
María Rocío Ruiz Calzado
}

Facultad de Enfermería. Universidad de Córdoba

\section{Resumen}

En los pacientes con enfermedad renal crónica portadores de un trasplante renal, la adherencia al tratamiento es crucial para prevenir el rechazo, pérdida del injerto, y la morbilidad adicional.

Objetivo: conocer la producción científica actual de la adherencia al tratamiento de pacientes trasplantados de riñón y las variables que pueden influir.

Método: Se realizó una búsqueda en las bases de datos de Scielo, PubMed, The Chocrane, Science Direct y Google académico. Se incluyeron artículos científicos escritos en inglés y español. Se analizaron los artículos que trataban la adherencia al tratamiento inmunosupresor de pacientes trasplantados de riñón, excluyendo los pacientes pediátricos.

Resultados: La adherencia al tratamiento inmunosupresor por parte de estos pacientes es relativamente baja debido a la influencia de varios factores. La complejidad del tratamiento y el tiempo postrasplante, tienen una importante correlación negativa con la adherencia. El olvido u otras ocupaciones, se relaciona de forma significativa con la no adherencia no intencional por parte de los pacientes. Los grupos de edad con menos adherencia son los más jóvenes seguidos de los más mayores, y los más

\author{
Correspondencia: \\ María Rocío Ruíz Calzado \\ Facultad de Enfermería \\ Universidad de Córdoba \\ Avda. Menéndez Pidal s/n \\ 14005. Córdoba \\ E-mail: rocioruizcalzado@gmail.com
}

adherentes los adultos. En algunos países, la falta de adherencia se debe a que el elevado coste del tratamiento inmunosupresor corre por cuente del paciente.

Por otro lado, las variables que no parecen influir de forma concluyente, son: el estado civil o modo de vida, efectos secundarios, nivel socioeconómico y educativo, situación laboral, raza, creencias en la necesidad y factores psicológicos. Por último, la variable de género es claramente la única que no influye sobre la falta de adherencia, ya que una mayoría de estudios demuestran que no influyen frente a solo dos que consideran que los varones son los menos adherentes.

Conclusiones: los pacientes trasplantados de riñón presentan altos porcentajes de falta de adherencia al tratamiento, estando relacionados fundamentalmente la complejidad del tratamiento, el tiempo postrasplante, el olvido u otras ocupaciones y la edad (jóvenes y mayores).

\section{PALABRAS CLAVE:}

- TRASPLANTE RENAL ADHERENCIA AL TRATAMIENTO

- TRATAMIENTO INMUNOSUPRESOR

\section{Therapeutic adherence in kidney transplant pa- tients}

\section{Abstract}

In patients with chronic kidney disease who have a kidney transplant, adherence to treatment is crucial to prevent rejection, graft loss and additional morbility. 
Objective: to know current scientific production on adherence to treatment by kidney transplant patients and the variables that can affect it.

Method: A search was conducted in the databases of Scielo, PubMed, The Chocrane, Science Direct and Google Scholar. Scientific articles written in English and Spanish were included. Articles that dealt with adherence to immunosuppressive treatment by kidney transplant patients were analysed, excluding paediatric patients.

Results: Adherence to immunosuppressive treatment by these patients is relatively low due to the influence of several factors. The complexity of the treatment and time after the transplant have an important negative correlation to adherence. Forgetfulness or other occupations are related significantly to unintentional non-adherence by patients. The age groups with the lowest adherence are the youngest followed by the most elderly, while the most adherent are adults. In some countries, the lack of adherence is due to the fact that the high cost of the immunosuppressive treatment has to be paid by the patient.

On the other hand, variables that do not appear to have a conclusive influence are: marital status or lifestyle, side effects, socio-economic and educational level, job situation, race, beliefs about their necessity and psychological factors. Finally, the variable of gender is clearly the only one that does not affect lack of adherence, as most studies show that gender does not influence adherence compared to only two that consider that men are less adherent.

Conclusions: kidney transplant patients present higher percentages of lack of adherence to treatment, fundamentally related to the complexity of the treatment, the time since the transplant, forgetfulness, or other occupations and age (young and elderly).

\section{KEY WORDS:}

- KIDNEY TRANSPLANT TREATMENT ADHERENCE

- IMMUNOSUPPRESSIVE TREATMENT

\section{Introducción}

El trasplante renal (TR) es el tratamiento de elección para los pacientes con enfermedad renal terminal (ERT) ya que se asocia con una mayor supervivencia del paciente, una mejor calidad de vida y un coste menor, que el tratamiento sustitutivo con diálisis ${ }^{1-5}$. Durante las últimas décadas, este tratamiento se ha generalizado de manera progresiva entre un mayor número de pacientes, de tal forma que en nuestro país alrededor de la mitad de los pacientes con IRCT son portadores de un TR funcionante ${ }^{6}$. Los registros existentes en distintos países confirman una progresiva mejoría de los resultados del TR a corto plazo. Actualmente, la incidencia de rechazo agudo es menos del $15 \%$ y la supervivencia del injerto es mayor de $90 \%$ al año del TR. En cambio, la evaluación de los resultados a largo plazo ha sido difícil de interpretar, ya que se han reportado informaciones contradictorias ${ }^{7,8}$.

EI TR no presenta las limitaciones diarias que puede presentar la diálisis, pero requiere una estricta adherencia a un régimen terapéutico como es el tratamiento inmunosupresor. Esto es esencial para la supervivencia del injerto renal. La no adherencia a la medicación se asocia con pobres resultados clínicos, tales como aumento de la incidencia de rechazo (agudo o crónico) y, en consecuencia acortando la supervivencia del injerto renal9-12.

La pérdida del injerto renal como consecuencia de un inadecuado cumplimiento terapéutico fue descrito por primera vez en 1975 por Owen y Cols. ${ }^{13}$, señalando una prevalencia del $2 \%$ de abandono voluntario de los inmunosupresores. Diversos estudios desarrollados en los años siguientes mostraron prevalencias de incumplimiento similares, oscilando entre el $2-4.7 \%$ en pacientes con distintos regímenes terapéuticos ${ }^{14}$. Sin embargo estudios más recientes han demostrado incrementos significativos en la prevalencia, con cifras que oscilan entre el 18-20\%, representando, en algunas series, la tercera causa de pérdida del injerto ${ }^{15}$.

Aunque se ha producido una notable mejora en la supervivencia del injerto gracias a los avances médicoquirúrgicos, a la profilaxis de las enfermedades infecciosas y el desarrollo de medicamentos inmunosupresores (tales como tacrolimus y micofenolato motefil) ${ }^{16}$; la falta de adherencia a los fármacos inmusupresores sigue siendo uno de los de las causas más importantes de pérdida del injerto renal ${ }^{16-18}$. Por tanto, la adherencia al tratamiento en los pacientes con ERT que reciben 
un trasplante renal es vital, pues de esto depende la supervivencia del injerto ${ }^{19}$. No obstante, los resultados publicados son poco homogéneos y variables, por lo que nos planteamos profundizar en el tema.

El objetivo por tanto, del presente estudio fue conocer la producción científica acerca de la falta de adherencia o cumplimiento terapéutico en los pacientes trasplantados de riñón.

\section{Material y métodos}

El diseño empleado es el de un estudio de revisión bibliográfica. Para ello, se hizo una búsqueda exhaustiva en diversas bases de datos con el objetivo de recopilar la mayor producción posible disponible con respecto al tema a tratar. Aunque en un principio se pensó en recopilar artículos recientes comprendidos entre 20082012, más tarde se amplió este periodo de tiempo para rescatar artículos muy interesantes acerca de la adherencia al tratamiento que habían sido publicados bastantes años atrás los cuales no podíamos obviar (2004-2012). Aunque si reservamos los artículos más recientes para hablar acerca de ellos en los resultados y la discusión por la actualidad de sus datos.

Las bases de datos empleadas fueron: Scielo, Pubmed, The Cochrane, Science Direct y Google Académico. EI periodo de consulta de las bases de datos tuvo lugar desde el 1 de Marzo de 2013 hasta el 1 de Junio de 2013.

El método de búsqueda consistió en utilizar como palabras clave en las diversas bases de datos: adherencia al tratamiento (adherence to treatment); trasplante renal (kidney trasplant) unidos dichos términos por los conectores boleanos "and" y "or", y empleando los distintos descriptores en singular, plural y que los descriptores presentes en el resumen, título del artículo y, si la base de datos lo presenta, en palabras clave.

Los criterios de inclusión fueron:

- Estudios en los cuales se tratara la adherencia al tratamiento prescrito de pacientes trasplantados adultos.

- Artículos originales.

- Hemos aceptado revisiones y metaanálisis.

Los criterios de exclusión fueron:

- Pacientes trasplantados renales pediátricos.

- Aquellos que no presentaran resultados.
- Solo se aceptaron artículos escritos en inglés o en castellano, puesto que son las dos lenguas con mayor producción científica y que el revisor conoce.

Al inicio de la búsqueda no se incluyeron los criterios de inclusión/exclusión en las diversas bases de datos para conocer la cantidad de producción científica existente en el tema y obtener mayor conocimiento en el tema. Después, si colocamos dichos criterios en las bases de datos para obtener sobre todo artículos más recientes.

Posteriormente se procedió a la lectura de los resúmenes y títulos de todos los artículos, teniendo en cuenta los criterios de inclusión y exclusión, con el objetivo de eliminar los que no tuvieran relación con el tema tratado y eliminar los que estuviesen repetidos.

Tras esta segunda selección o proceso de cribado, se procedió a recopilar toda la producción científica y eliminar aquellos que anteriormente habían pasado el cribado pero que se dudaba de su relación con el tema a tratar, revisando el artículo completo. También se excluyeron en este apartado los artículos con muestras demasiadas pequeñas.

\section{Resultados}

\section{Selección de estudios preliminar}

En función de las distintas bases de datos el método de búsqueda varió, empleando distintos métodos. De un total de 166, descartamos 30 y preseleccionamos 136 artículos. (Tabla 1).

\begin{tabular}{|l|c|c|c|c|c|} 
& PubMed & $\begin{array}{c}\text { The } \\
\text { Chocrane }\end{array}$ & Scielo & $\begin{array}{c}\text { Science } \\
\text { Direct }\end{array}$ & $\begin{array}{c}\text { Academic } \\
\text { Google }\end{array}$ \\
\hline $\begin{array}{l}\text { Artículos } \\
\text { encontrados }\end{array}$ & 102 & 5 & 18 & 13 & 28 \\
\hline $\begin{array}{l}\text { Artículos } \\
\text { preseleccio- } \\
\text { nados }\end{array}$ & 81 & 5 & 18 & 11 & 21 \\
\hline
\end{tabular}

Tabla I.

En total, de los 136 documentos preseleccionados de todas las bases de datos, tras eliminar los repetidos (17), se quedaran 109 artículos que cumplían con los criterios de inclusión/exclusión establecidos en este estudio de revisión.

En la segunda revisión tras hacer una lectura completa de todos los artículos volvimos a excluir algunos más, 
[ María Rocío Ruiz Calzado, et al ]

La adherencia terapéutica en el paciente trasplantado renal

porque no encajaban del todo con el objetivo de nuestra revisión, dejando así finalmente los que aparecen en la bibliografía, quedándonos finalmente con los artículos seleccionados en la Tabla II.

\begin{tabular}{|c|c|c|c|c|}
\hline Autor, año, país & Muestra & $\begin{array}{l}\% \text { de no } \\
\text { adherencia }\end{array}$ & Diseño del estudio & Variable estudiada \\
\hline $\begin{array}{c}\text { Israni AK, Weng FL, Cen Y-Y, Joffe } \\
\text { M, Kamoun M, Feldman HI. } 2011 . \\
\text { USA }\end{array}$ & 243 & $\begin{array}{c}30(12 \%) \\
-<\text { del } 50 \% \text { de } \\
\text { adherencia }\end{array}$ & Cohorte prospectivo & $\begin{array}{c}\text { Raza y relación directa entre el } \\
\text { incumplimiento terapéutico y la pérdida } \\
\text { del injerto o rechazo agudo. }\end{array}$ \\
\hline $\begin{array}{c}\text { G. Germani, } \\
\text { S. Lazzaro, } \\
\text { F. Gnoato, et al. 2011. Italy }{ }^{23} \text {. }\end{array}$ & 218 & $379 \%$ & $\begin{array}{l}\text { Observacional } \\
\text { longitudinal }\end{array}$ & $\begin{array}{l}\text { Edad, tiempo postrasplante, estado civil, } \\
\text { empleado, nivel educativo y género. }\end{array}$ \\
\hline $\begin{array}{l}\text { Janet a. Butler, Paul Roderick, Mark } \\
\text { mullee, Juan C. Mason, and Robert c. } \\
\text { Peveler. 2004. USA }{ }^{38} \text {. }\end{array}$ & 325 estudios & $\begin{array}{l}\text { Media de } 22 \% \\
(18 \%-26 \%)\end{array}$ & Revisión sistemática & $\begin{array}{l}\text { Olvido. Relación directa entre el } \\
\text { cumplimiento y la pérdida del injerto o } \\
\text { rechazo agudo. }\end{array}$ \\
\hline $\begin{array}{c}\text { C.-Y. Cheng, B.Y.-J. Lin, K.-H. Chang, } \\
\text { K.-H. Shu, and M.-J. Wu. } 2012 . \\
\text { USA }\end{array}$ & 412 & $21 \times 4 \%$ & $\begin{array}{l}\text { Prospectivo, } \\
\text { controlado. }\end{array}$ & $\begin{array}{l}\text { Género, edad, nivel educativo, } \\
\text { complejidad del tratamiento y relación } \\
\text { directa entre el cumplimiento y la } \\
\text { pérdida del injerto o rechazo agudo. }\end{array}$ \\
\hline $\begin{array}{l}\text { Cukor D, Rosenthal DS, Jindal RM, } \\
\text { Brown CD, Kimmel PL. 2009. USA }\end{array}$ & 159 & $19 \%(n=29)$ & $\begin{array}{l}\text { Observacional } \\
\text { analítico }\end{array}$ & Raza, género y factores psicológicos. \\
\hline $\begin{array}{c}\text { Chisholm MA, Williamson GM, Lance } \\
\text { CE, Mulloy LL. 2007. USA }{ }^{36} \text {. }\end{array}$ & 158 & $27 \%$ & $\begin{array}{l}\text { Observacional } \\
\text { descriptivo }\end{array}$ & Edad, factores psicológicos. \\
\hline $\begin{array}{c}\text { Clifford S, Barber N, Horne R. } 2008 . \\
\text { UK }\end{array}$ & 239 & $30 \%(n=67)$ & Estudio transversal & $\begin{array}{l}\text { Edad, raza, creencias en la necesidad y } \\
\text { género. }\end{array}$ \\
\hline $\begin{array}{l}\text { Konstadina Griva, Ph.D. \&Andrew } \\
\text { Davenport, FDRC \& } \\
\text { Michael Harrison, FDRC \&Stanton P. } \\
\text { Newman, Ph.D. 2012. UK }{ }^{31} \text {. }\end{array}$ & 218 & $254 \%$ & Estudio transversal & $\begin{array}{l}\text { Edad, situación laboral, nivel } \\
\text { educativo, género, estado civil, } \\
\text { factores psicológicos, complejidad del } \\
\text { tratamiento, olvido. }\end{array}$ \\
\hline $\begin{array}{c}\text { Weng FL, Israni AK, Joffe M M, Hoy } \\
\text { T, Gaughan CA, Newman M, Abrams } \\
\text { JD, Kamoun M, Rosas SE, Mange KC, } \\
\text { Strom BL, Brayman KL, Feldman HI.. } \\
\text { 2005. USA }{ }^{41} \text {. }\end{array}$ & 278 & $\begin{array}{c}(13,7 \%) \\
-<\text { del } 50 \% \text { de } \\
\text { adherencia }\end{array}$ & Cohorte prospectivo & Raza. \\
\hline $\begin{array}{l}\text { Pinsky BW, Takemoto SK, Lentine } \\
\text { KL, Burroughs TE, Schnitzler MA, } \\
\text { Salvalaggio PR. 2009. USA }{ }^{22} \text {. }\end{array}$ & 15525 & $23,1 \%(n=2589)$ & $\begin{array}{l}\text { Estudio de cohorte } \\
\text { prospectivo }\end{array}$ & Edad y efectos secundarios. \\
\hline $\begin{array}{l}\text { Thomas E. Nevinsl, William Thomas. } \\
\text { 2009. USA }{ }^{33 .}\end{array}$ & 137 & $16,78 \%(n=23)$ & $\begin{array}{l}\text { Observacional } \\
\text { lontitudinal } \\
\text { prospectivo }\end{array}$ & $\begin{array}{l}\text { Edad, raza, relación entre no } \\
\text { adherencia y pérdida del aloinjeto. }\end{array}$ \\
\hline $\begin{array}{l}\text { Marie A.Chisholms-Bunns, W. } \\
\text { Jaqueline Kwong, Laura L Mulloy, } \\
\text { Christina A. Spivey. 2008. USA }{ }^{35 .}\end{array}$ & 70 & $12,9 \%$ & $\begin{array}{l}\text { Observacional } \\
\text { analitico }\end{array}$ & Edad y tiempo postrasplante. \\
\hline $\begin{array}{c}\text { Roger W. Evans, William H. } \\
\text { Applegate, David M. Briscoe, David } \\
\text { J. Cohen, } \\
\text { Christopher C. Rorick, Barbara T. } \\
\text { Murphy, and Joren C. Madsen. } 2010 . \\
\text { USA }{ }^{42} \text {. }\end{array}$ & 254 & $10 \%$ & Estudio descriptivo & $\begin{array}{l}\text { Adherencia y costo del tratamiento y } \\
\text { pérdida del injerto como consecuencia } \\
\text { de los costos del tratamiento. }\end{array}$ \\
\hline $\begin{array}{c}\text { Couzi L, Moulin B, Morin MP, Albano } \\
\text { L, Godin M, Barrou B, Alamartine } \\
\text { E, Morelon E, Girardot-Seguin S, } \\
\text { Mendes L, Misdrahi D, Cassuto E, } \\
\text { Merville P.. 2013. } \text { France }^{20} \text {. }\end{array}$ & 312 & $\begin{array}{c}17.3 \%: 3 \text { meses } \\
\text { postrasplante } \\
24.1 \%: 6 \text { meses } \\
\text { postrasplante } \\
30.7 \%: 12 \text { meses } \\
\text { postrasplante } \\
34.6 \%: 24 \text { meses } \\
\text { postrasplante }\end{array}$ & $\begin{array}{l}\text { Estudio de cohorte } \\
\text { prospectivo }\end{array}$ & $\begin{array}{l}\text { Complejidad del tratamiento, tiempo } \\
\text { postrasplante, factores psicológicos, } \\
\text { edad, género, educación, empleo, } \\
\text { efectos secundarios y estado civil. }\end{array}$ \\
\hline $\begin{array}{c}\text { Brahm MM, Manfro RC, Mello } \\
\text { D, Cioato S, Gonçalves LF. } 2012 . \\
\text { Brazil }^{34} \text {. }\end{array}$ & 288 & $58,7 \%(n=168)$ & $\begin{array}{l}\text { Estudio descriptivo } \\
\text { Transversal }\end{array}$ & Edad y situación laboral. \\
\hline $\begin{array}{c}\text { Dharancy S, Giral M, Tetaz R, Fatras } \\
\text { M, Dubel L, Pageaux GP.. } 2012 . \\
\text { France }^{32} \text {. }\end{array}$ & 381 & $73 \%$ & $\begin{array}{l}\text { Observacional } \\
\text { de cohortes }\end{array}$ & $\begin{array}{l}\text { Edad, tiempo postrasplante, género y } \\
\text { complejidad del tratamiento. }\end{array}$ \\
\hline
\end{tabular}




\begin{tabular}{|c|c|c|c|c|}
\hline Autor, año, país & Muestra & $\begin{array}{l}\% \text { de no } \\
\text { adherencia }\end{array}$ & Diseño del estudio & Variable estudiada \\
\hline $\begin{array}{c}\text { Chisholm-Burns M, Pinsky B, Parker } \\
\text { G, Johnson P, Arcona S, Buzinec P, } \\
\text { Chakravarti P, Good M, Cooper M.. } \\
\text { 2012. USA }{ }^{27} \text {. }\end{array}$ & 512 & $34.5 \%(n=177)$ & $\begin{array}{l}\text { Estudio descriptivo } \\
\text { transversal }\end{array}$ & $\begin{array}{l}\text { Olvido, edad, creencias en la necesidad, } \\
\text { educación, raza, género, estado civil, } \\
\text { situación laboral, tiempo postrasplante. }\end{array}$ \\
\hline $\begin{array}{l}\text { Russell CL, Cetingok M, Hamburger } \\
\text { KQ, Owens S, Thompson D, Hathaway } \\
\text { D, Winsett RP, Conn VS, Madsen R, } \\
\text { Sitler L, Wakefield MR. 2010. USA }\end{array}$ & 37 & $86 \%$ & $\begin{array}{l}\text { Descriptivo } \\
\text { longitudinal }\end{array}$ & $\begin{array}{l}\text { Edad, raza, estado civil, situación } \\
\text { laboral, factores psicológicos, creencias } \\
\text { en la necesidad, tiempo postrasplante, } \\
\text { género y efectos secundarios. }\end{array}$ \\
\hline $\begin{array}{l}\text { Schmid-Mohler G, Thut MP, Wüthrich } \\
\text { RP, Denhaerynck K, De Geest S. } \\
\text { 2010. Zurich }{ }^{39} \text {. }\end{array}$ & 114 & $237 \%$ & $\begin{array}{l}\text { Descriptivo } \\
\text { Transversal }\end{array}$ & Olvido \\
\hline $\begin{array}{c}\text { Ichimaru N, Kakuta Y, Abe T, Okumi } \\
\text { M, Imamura R, Isaka Y, Nonomura } \\
\text { N, Kojima Y, Okuyama A, Takahara S. } \\
\text { 2008. Japan }{ }^{37} \text {. }\end{array}$ & 507 & $358 \%$ & $\begin{array}{l}\text { Observacional } \\
\text { descriptivo }\end{array}$ & Tiempo postrasplante \\
\hline $\begin{array}{l}\text { Gremigni P, Bacchi F, Turrini C, } \\
\text { Cappelli G, Albertazzi A, Bitti PE. } \\
\text { 2007. Italy }{ }^{28} \text {. }\end{array}$ & 34 & $24 \%$ & $\begin{array}{l}\text { Descriptivo } \\
\text { Trasversal }\end{array}$ & Edad, género, factores psicológicos \\
\hline $\begin{array}{c}\text { Russell CL, Conn VS, Ashbaugh C, } \\
\text { Madsen R, Hayes K, Ross G.. } 2010 . \\
\text { USA }^{29} \text {. }\end{array}$ & 44 & $25 \%$ & $\begin{array}{l}\text { Prospectivo, } \\
\text { descriptivo }\end{array}$ & $\begin{array}{c}\text { Edad, género, raza, estado civil, empleo, } \\
\text { tiempo postrasplante. }\end{array}$ \\
\hline $\begin{array}{c}\text { Chisholm MA, Lance CE, Mulloy LL. } \\
\text { 2005. USA }{ }^{30} \text {. }\end{array}$ & 137 & $35 \%(n=48 \%)$ & $\begin{array}{l}\text { Observacional } \\
\text { descriptivo }\end{array}$ & $\begin{array}{c}\text { Género, edad, nivel socioeconómico, } \\
\text { tiempo postrasplante. }\end{array}$ \\
\hline
\end{tabular}

Tabla II. Clasificación de todos los artículos seleccionados para el análisis de prevalencia de falta de adherencia al tratamiento

De todos los artículos incluidos en la tabla, ninguno presentaba limitaciones, tan solo uno de ellos de Dharancy S, Giral M, Tetaz R, Fatras M, Dubel L, Pageaux GP32 que destaco varias limitaciones de su estúdio como son; en cuanto a la recogida de datos y no todos los pacientes podían ser analizados en este estudio. Además, éste estudio, sólo incluyó una visita, por lo tanto, la adhesión no puede ser evaluada con clínica de resultados en cualquier otra medición de seguimiento.

En la siguientes tablas (tablas IIIa,b,c) se representan los factores asociados a la adherencia.

\begin{tabular}{|c|c|c|}
\hline & No influye & Sí influye \\
\hline \multirow{2}{*}{$\begin{array}{l}\text { Efectos secundarios o efectos } \\
\text { adversos }\end{array}$} & $n=31220$ & $\begin{array}{c}n=1552522 \text { OR } 1.20 \\
p=0.0001\end{array}$ \\
\hline & $\begin{array}{l}n=3721 \\
p=0,43\end{array}$ & \\
\hline $\begin{array}{l}\text { Adherencia y costo del trata- } \\
\text { miento y pérdida del injerto } \\
\text { como consecuencia de los cos- } \\
\text { tos del tratamiento }\end{array}$ & & $\begin{array}{l}\mathrm{n}=25442 \text {. A los } 3 \text { años postrasplante, Medicare no cubre los fármacos y la adherencia } \\
\text { disminuye. } \\
\text { Dificultad para pagar los medicamentos inmunosupresores } 88 \% \text {. } \\
\text { Muerto pérdidas de injerto debido a la falta de adherencia relacionada con los costos de la } \\
\text { medicación inmunosupresora } 50.7 \%\end{array}$ \\
\hline
\end{tabular}

Tabla III a. Factores asociados a la adherencia. Efectos secundarios o efectos adversos.- Adherencia y costo del tratamiento y pérdida del injerto como consecuencia de los costos del tratamiento.

\begin{tabular}{|c|c|c|c|c|c|}
\hline & No influye & Sí influye & Jóvenes & Adultos & Mayores \\
\hline \multirow{6}{*}{ Edad } & $n=412^{24}$ & $n=158^{36}$ & & & $\begin{array}{l}\text { Menos adherentes } \\
(p=0.01)\end{array}$ \\
\hline & $n=312^{20}$ & $n=218^{31}$ & $\begin{array}{l}\text { Menos adherentes ( } r \text { 0.19; } \\
\text { p 0.006) }\end{array}$ & & \\
\hline & $n=37^{21}$ & $n=137^{33}$ & $\begin{array}{l}\text { Menos adherentes ( } p \\
0.047 \text { ) }\end{array}$ & & \\
\hline & $n=239^{26}$ & $\mathrm{n}=70^{35}$ & & $\begin{array}{l}\text { Más adherentes } \\
\text { (de } 18 \text { a } 60 \text { años) }\end{array}$ & $\begin{array}{l}\text { Menos adherentes ( } 60 \text { o más } \\
\text { años) }\end{array}$ \\
\hline & $n=218^{23}$ & $n=288^{34}$ & $\begin{array}{l}\text { Menos adherentes } \\
(p 0.011)\end{array}$ & & \\
\hline & $n=34^{29}$ & $n=512^{27}$ & Menos adherentes & Más adherentes & \\
\hline
\end{tabular}


[ María Rocío Ruiz Calzado, et al ]

La adherencia terapéutica en el paciente trasplantado renal

\begin{tabular}{|c|c|c|c|c|c|}
\hline \multirow{4}{*}{ Edad } & & $n=34^{28}$ & $\begin{array}{l}\text { Menos adherentes } \\
(p=0.02)\end{array}$ & & \\
\hline & & $n=15525^{22}$ & Menos adherentes & Más adherentes & \\
\hline & & $n=381^{32}$ & & $\begin{array}{l}\text { Más adherentes } \\
(p=0.02)\end{array}$ & \\
\hline & & $n=137^{30}$ & $\begin{array}{l}\text { Más adherentes } \\
(p=0.001)\end{array}$ & & \\
\hline & No influyen & Si influyen & $<$ de 1 año & De 1 a 4 años & $>$ de 4 años \\
\hline \multirow{6}{*}{$\begin{array}{c}\text { Tiempo } \\
\text { Postrasplante }\end{array}$} & $n=512^{27}$ & $n=218^{23}$ & & & $\begin{array}{l}\text { Menos adherentes } \\
(p=0.02)\end{array}$ \\
\hline & $n=37^{21}$ & $\mathrm{n}=70^{35}$ & & & $\begin{array}{l}\text { Menos adherentes } \\
(p<0.01) \\
0.3 \% \text { menos adherencia por cada } \\
\text { año más. }\end{array}$ \\
\hline & $n=34^{29}$ & $n=381^{32}$ & & $\begin{array}{l}\text { Más adherencia } \\
35 \cdot 2 \%\end{array}$ & $\begin{array}{l}\text { Menos adherencia } \\
22 \% \text {. }\end{array}$ \\
\hline & & $n=312^{20}$ & No adherencia $30.7 \%$ & $\begin{array}{l}\text { Menos adherentes. No adhe- } \\
\text { rencia } 34.6 \% \text {. }\end{array}$ & \\
\hline & & $n=507^{37}$ & & & $\begin{array}{l}\text { Menos adherentes. Adherencia } \\
\text { disminuye cuando aumenta el } \\
\text { tiempo. }\end{array}$ \\
\hline & & $n=137^{30}$ & & $\begin{array}{l}\text { Menos adherencia que } \\
\text { durante el primer año } \\
(p=0.017)\end{array}$ & \\
\hline
\end{tabular}

Tabla III b. Factores asociados a la adherencia. Edad. Tiempo postrasplante.

\begin{tabular}{|c|c|c|c|c|}
\hline & No influyen & Sí influyen & Blancos & Negros \\
\hline \multirow{7}{*}{ Raza } & $n=159^{25}$ & $n=278^{41}$ & & $\begin{array}{l}\text { Menos adherentes } \\
\text { ( OR: } 0 ` 43 \text { IC: } 0.26 \text { a 0.72. P 0.001) }\end{array}$ \\
\hline & $n=239^{26}$ & $n=137^{33}$ & & $\begin{array}{l}\text { Menos adherentes } \\
(p=0.012)\end{array}$ \\
\hline & $n=512^{27}$ & $n=243^{40}$ & & $\begin{array}{l}\text { Menos adherentes } \\
(p=0,019)\end{array}$ \\
\hline & $\mathrm{n}=37^{21}$ & & & \\
\hline & $n=34^{29}$ & & & \\
\hline & $\mathrm{n}=137^{30}$ & & & \\
\hline & No influyen & Sí influyen & Complejo & No complejo \\
\hline \multirow{5}{*}{$\begin{array}{l}\text { Complejidad del } \\
\text { tratamiento }\end{array}$} & & $n=412^{24}$ & Menos adherentes & \\
\hline & & $n=312^{20}$ & & Menos adherentes \\
\hline & & $n=218^{31}$ & $\begin{array}{l}\text { Menos adherentes } \\
\left(13^{\prime} 8 \%\right)\end{array}$ & \\
\hline & & $n=381^{32}$ & $\begin{array}{l}\text { Menos adherencia } \\
(24 \%)\end{array}$ & Más adherencia (45\%) \\
\hline & No influye & Sí influye & Soltero o solo & Casado o en pareja \\
\hline \multirow{4}{*}{$\begin{array}{l}\text { Estado civil o modo } \\
\text { de vida }\end{array}$} & $\mathrm{n}=512^{27}$ & $\mathrm{n}=218^{31}$ & & Más adherentes $\left(p=0^{`} 032\right)$ \\
\hline & $\begin{array}{c}n=37^{21} \\
(p=0.41)\end{array}$ & $n=312^{20}$ & $\begin{array}{l}\text { Menos adherentes } \\
(30.8 \%)\end{array}$ & \\
\hline & $\mathrm{n}=218^{23}$ & & & \\
\hline & $\begin{array}{c}n=34^{29} \\
(p=0.47)\end{array}$ & & & \\
\hline
\end{tabular}




\begin{tabular}{|c|c|c|c|c|}
\hline & No influyen & Sí influyen & Alto & Bajo \\
\hline \multirow{5}{*}{$\begin{array}{l}\text { Nivel socioeconómico } \\
\text { y educativo }\end{array}$} & $n=412^{24}$ & $n=218^{31}$ & & $\begin{array}{l}\text { Menos adherentes } \\
\text { (p 0.001) }\end{array}$ \\
\hline & $\mathrm{n}=312^{20}$ & $\mathrm{n}=137^{30}$ & & $\begin{array}{l}\text { Más adherentes } \\
(p=0.001)\end{array}$ \\
\hline & $\mathrm{n}=512^{27}$ & & & \\
\hline & $\mathrm{n}=218^{23}$ & & & \\
\hline & No influye & Sí influye & Empleado & Desempleado \\
\hline \multirow{5}{*}{ Situación laboral } & $n=312^{20}$ & $\mathrm{n}=218^{31}$ & $\begin{array}{l}\text { Menos adherentes } \\
\left(n^{\circ} 12156 \%\right) \\
\text { (P 0.010) }\end{array}$ & \\
\hline & $\mathrm{n}=512^{27}$ & $n=288^{34}$ & $\begin{array}{l}\text { Menos adherentes } \\
\text { ( } p \text { 0.008) }\end{array}$ & \\
\hline & $n=37^{21}$ & & & \\
\hline & $\mathrm{n}=218^{23}$ & & & \\
\hline & $n=34^{29}$ & & & \\
\hline
\end{tabular}

Tabla III c. Factores asociados a la adherencia. Raza.- Complejidad del tratamiento.- Estado civil o modo de vida.- Nivel socioeconómico.Situación laboral.

En la tablas IV a, b, se recoge la prevalencia según variable y adherencia.

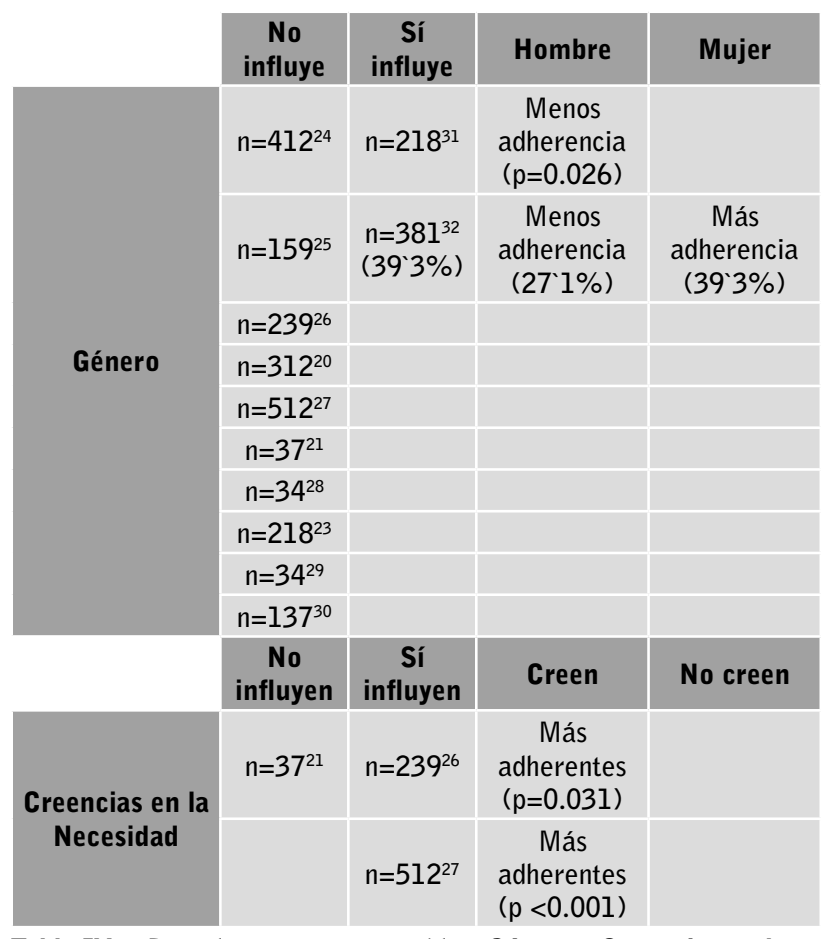

Tabla IV a. Prevalencia según variables. Género.- Creencias en la necesidad.

\section{Descripción de los resultados}

\section{- Variables específicas}

\section{Efectos secundarios o efectos adversos}

No está establecido la influencia que los efectos secundarios que producen los fármacos inmunosupresores condicionen la falta de adherencia al tratamiento ${ }^{20,21}$. Sin embargo, otro

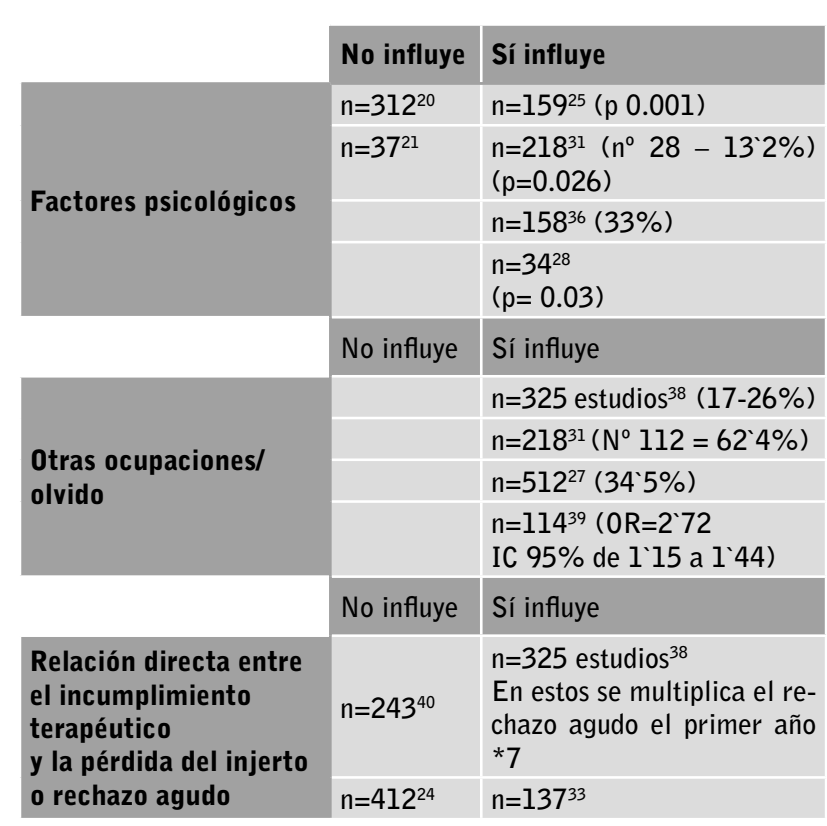

Tabla IV b. Prevalencia según variables.- Factores psicológicos.Otras ocupaciones/olvido.- Relación directa entre el incumplimiento terapéutico y la pérdida del injerto o rechazo agudo.

estudio encuentra una relación directa entre aparición de efectos adversos y falta de adherencia al tratamiento 22 .

\section{Género}

Muchos son los estudios que muestran que no hay diferencia significativa en cuanto al género en la adherencia al tratamiento $20,21,23-30$. Tan solo dos estudios encuentran diferencias significativas, 
concluyendo que los pacientes menos adherentes fueron hombres ${ }^{31,32}$.

\section{Complejidad del tratamiento}

La mayoría de los estudios analizados encuentran que el tratamiento más complejo disminuye la adherencia por parte de los pacientes ${ }^{24,31,32}$. Sin embargo, otro estudio encuentra relación entre el tratamiento menos complejo y la disminución de la adherencia de los pacientes ${ }^{20}$.

\section{Edad}

La edad predice patrones de adhesión, siendo los más jóvenes los menos adherentes ${ }^{22,27,28,33,33,34}$, aunque otro estudio considera que los pacientes más adherentes son los jóvenes ${ }^{30}$. Otros autores, comunican que el aumento de edad de los pacientes sin llegar a la ancianidad, es decir, adultos, se asocia con un mejor cumplimiento $22,27,32,35$. Los pacientes mayores presentan, en general, peor adherencia, ${ }^{35,36}$.

En contra de estos resultados, seis estudios no encuentran relación entre la edad y la falta de adherencia $20,21,23,24,26,29$.

\section{Tiempo postrasplante}

Numerosos estudios, muestran que con más tiempo de evolución del trasplante, la adherencia al tratamiento inmunosupresor por parte de los pacientes trasplantados disminuye. Algunos consideran que donde realmente desciende de manera considerable la adherencia al tratamiento

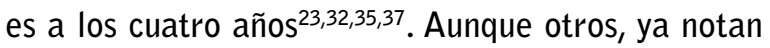
un descenso considerable a los dos años ${ }^{20,30}$.

Por el contrario, existen otros artículos que no encuentran que esta variable influya en la adherencia ${ }^{21,27,29}$.

\section{Estado civil o modo de vida}

Son más estudios los que demuestran que esta variable no incide en la adherencia ${ }^{21,23,27,29}$, que aquellos que sí encuentran diferencias significativas . Tan solo hemos encontrado dos estudios que consideran que el vivir solo o estar soltero hace que la adherencia por parte de los pacientes disminuya20,31.

\section{Factores psicológicos}

Los factores psicológicos como pueden ser el estrés y la ansiedad, parecen influir potencialmente de forma negativa en la adherencia al tratamiento $25,28,31,36$. Por el contrario, tan solo dos artículos ${ }^{20,21}$ demuestran que esta variable no influye.

\section{Situación laboral}

La mayoría de artículos encontrados que analizan esta variable consideran que la situación laboral no influye $20,21,23,27,29$. Tan solo algunos artículos ${ }^{31,34}$ encuentran que los pacientes que tienen un empleo, presentan menos adherencia que aquellos que no tienen una ocupación.

\section{Nivel socio económico y nivel educativo}

La mayoría de artículos estudiados consideran que esta variable no es influyente ${ }^{20,23,24,27}$. Hemos encontrado solo dos artículos que relacionan esta variable con la adherencia pero se contraponen, uno considera que los pacientes con niveles socioeconómicos más bajos presentan una menor adherencia al tratamiento inmunosupresor tras el trasplante ${ }^{31}$. Mientras que, por el contrario, otro estudio considera que los pacientes con niveles socioeconómicos más bajos presentan una mayor adherencia ${ }^{30}$.

\section{Creencias en la necesidad y sentimiento de} eficacia del tratamiento

Son muy pocos los artículos que analizan esta variable, $\tan$ solo hemos encontrados tres, dos estudios encuentran que aquellos pacientes que consideran que el tratamiento es imprescindible para llegar a un buen estado de salud y creen que es totalmente efectivo son más adherentes que aquellos que no lo creen necesario ${ }^{26,27}$. Por el contrario un artículo $^{21}$ no encuentra diferencia significativa a la adherencia entre los pacientes que creen en la necesidad y entre los que no creen en la necesidad del tratamiento inmunosupresor.

\section{0tras ocupaciones/ olvido}

Esta variable influye en la falta de adherencia, el olvido muestra una falta de adherencia no intencional que es motivo en gran parte de la no adherencia de pacientes trasplantados $27,31,38,39$.

\section{Raza}

La mayoría de los estudios demuestran que esta variable no influye $\mathrm{e}^{21,25-27,29,30}$. Tan solo tres consideran que la raza influye en la adherencia, coincidiendo los tres en que los menos adherentes son los pacientes no caucásicos ${ }^{33,40,41}$. 


\section{Adherencia y costo del tratamiento}

Un artículo americano ${ }^{42}$ el cual analiza los costos del tratamiento inmunosupresor, encuentra que a los tres años postrasplante los pacientes experimentan una gran falta de adherencia al tratamiento inmunosupresor. Esto es debido a las deficiencias económicas que tienen para poder comprar dichos fármacos a los tres años postrasplante cuando el seguro ya no les cubre dichos medicamentos.

\section{Relación directa entre el incumplimiento terapéutico y la pérdida del injerto o rechazo agudo}

Hemos encontrado 6 artículos que analizan esta variable, tres de ellos encuentran relación con la falta de adherencia y otros tres no la encuentran. Algunos artículos relacionan de forma directa la falta de adherencia con rechazo agudo, la pérdida del injerto 0 incluso la muerte del paciente ${ }^{22,33,38}$. Mientras que otros $24,30,40$, consideran que no está relacionada la falta de adherencia con la pérdida del injerto u otros acontecimientos adversos. Cabe destacar un estudio ${ }^{22}$, el cual utiliza una muestra relevante de $\mathbf{1 5 . 5 2 5}$ pacientes y considera que la variable es influyente, ya que podría prevalecer de importancia sobre otros estudios que no encuentran relación alguna con la falta de adherencia.

\section{Discusión}

En esta revisión se ha puesto de manifiesto que la falta de adherencia al régimen terapéutico de los pacientes trasplantados de riñón está condicionada por múltiples factores. Según nuestra revisión, hemos observado que los efectos adversos o efectos secundarios son pocos estudiados. Tan solo tres artículos de nuestra búsqueda han incluido esta variable, de los cuales dos consideran que no influyen ${ }^{20,21}$. Por el contrario, tan solo un artículo22, aunque muy relevante debido a la gran muestra que ha utilizado encuentra relación con la falta de adherencia. Según este, parece ser que mientras más efectos adversos presentan los fármacos peor se sienten los pacientes y como consecuencia abandonan el tratamiento.

La variable de género no parece tener relación con la falta de adherencia de los pacientes $20,21,23-30$. Tan solo hemos encontrado dos estudios de toda nuestra revisión que consideren que el género masculino es el que menor adherencia presenta ${ }^{31,32}$. Por lo que podemos obtener una idea clara de la no influencia de esta variable en la adherencia al tratamiento de pacientes con TR.
Está bien documentado que la complejidad del tratamiento farmacológico influye en la adherencia al tratamiento inmunosupresor. De los artículos encontrados, la mayoría de ellos encuentra que el tratamiento más complejo es el que hace que los pacientes tengan una menor adherencia ${ }^{24,31,32}$. La reducción en la frecuencia de la dosificación ha dado lugar a mejoras generales en la adherencia a los inmunosupresores, satisfacción del paciente, calidad de vida y disminución de costos. Esto es debido a que los pacientes se cansan de tener que tomar tanta medicación y a diferentes horas y no llegan a adherirse nunca al tratamiento completo hasta que finalmente lo abandonan por completo. Por el contrario, tan solo uno de ellos ${ }^{20}$, considera que los tratamientos menos complejos hacen que los pacientes sean menos adherentes. Podríamos obtener una idea en claro y es que los tratamientos más complejos son los que impiden que la población de adhiera de forma correcta al tratamiento.

Hemos encontrado una gran cantidad de artículos que analizan la variable edad. La mayoría de estos artículos estudian el intervalo de pacientes jóvenes. La adherencia en cuanto a la edad se puede dividir en tres grupos. El primer grupo, los más jóvenes con escasa adherencia $22,27,28,31,33,34$, debido, por un lado, a que son menos responsables y no creen que sea necesario la toma del tratamiento. Aunque por el contrario, hemos encontrado un artículo que considera que los jóvenes son los más adherentes ${ }^{30}$. En segundo lugar, los adultos, son los pacientes que más adherencia presentan en comparación con los jóvenes y ancianos $22,27,32,35$, debido a que son más responsables que los jóvenes, lo creen necesario, y no tienen problemas cognitivos como pueden presentar los ancianos. Por último, los ancianos tienen una tasa alta de falta de adherencia ${ }^{35,36}$, la cual se justifica con los deterioros cognitivos y habilidades verbales. Menos adherencia tendrán aún si el tratamiento es complejo, sobre todo, en los ancianos viudos o solteros. Por contra, algunos artículos no encuentran relación entre la edad y la falta de adherencia ${ }^{20,21,23,24,26,29}$, aunque éstos son menos cantidad que los que sí encuentran relación. De esta variable podríamos sacar una idea bastante clara y es que los jóvenes son los menos adherentes, seguido de los ancianos, y los adultos el grupo de edad con más adherencia.

El tiempo postrasplante en los pacientes juega un papel importante. La mayoría de los estudios coinciden en que a medida que aumenta el tiempo postrasplante, la adherencia disminuye hasta llegar a dejar de tomar la medicación con el paso de los años $20,23,30,32,35,37$. La adhesión más alta se encuentra durante los dos primeros 
años después de haber recibido el aloinjerto, a partir de los dos años, se nota un descenso importante en la falta de cumplimiento terapéutico. Esto es debido, a que con el paso del tiempo, los pacientes se cansan de estar tomando cada día la medicación, por lo que comienzan a abandonar de forma gradual el tratamiento y al no sentir ningún tipo de cambio en su organismo creen que no es necesario y finalmente abandonan el tratamiento de forma completa. Por el contrario, tan solo hemos encontrado tres artículos que no encuentran relación de esta variable con la falta de adherencia ${ }^{21,27,29}$. por tanto, podríamos decir que el tiempo postrasplante influye, a medida que pasa el tiempo, la adherencia disminuye de forma gradual.

El estado civil o modo de vida parece no tener relación con la falta de adherencia según la mayoría de los artículos encontrados $21,23,27,29$. Tan solo dos consideran que el vivir solo o estar soltero disminuye la adherencia en estos pacientes ${ }^{20,31}$, según estos estudios, argumentan que los pacientes que viven solos no poseen la ayuda y el apoyo social de la persona que está a su lado y le recuerda la toma de la medicación. Vivir solo o en pareja no parece influir en la adherencia al tratamiento.

En cuanto a los factores psicológicos, como son el estrés y la ansiedad, hemos comprobado que están presentes en algunos pacientes TR e influyen potencialmente de forma negativa en la adherencia al tratamiento $25,28,31,36$. Estos estudios se basan en que las personas que tienen estrés 0 ansiedad se encuentran en un estado en el que no les importa nada, porque ya de por sí, se encuentran mal y no creen que la medicación les arregle nada. Por el contrario, dos artículos no encuentran relación de esta variable con la falta de adherencia ${ }^{20,21}$. No se puede concretar, por tanto, la relación de esta variable con la falta de adherencia, ya que tres artículos consideran que influye mientras que dos consideran que no influye.

Según la mayoría de los artículos encontrados el empleo parece no influir en la adherencia $20,21,23,27,29$. Tan solo dos estudios ${ }^{31,34}$ consideran que los pacientes empleados son los menos adherentes, debido a que cuando el trabajo ocupa la mayor parte de tu tiempo, hace que te olvides de la respectiva toma de la medicación. Además de las barreras que puede tener un trabajo para que se pueda llevar consigo la medicación y el paciente pueda dejar de trabajar para tomársela. En cuanto a esta variable podríamos decir que no influye en la adherencia al tratamiento inmunosupresor.
El nivel de socioeconómico y nivel educativo, es decir, el estatus social que ocupan los pacientes no determina patrones de adhesión según algunos artículos ${ }^{20,23,24,27}$. Tan solo dos artículos consideran que existe relación con la falta de adherencia, uno encuentra que los pacientes con bajo nivel socioeconómico y educativo son los menos adherentes ${ }^{31}$, mientras que, por el contrario, otro estudio encuentra que los pacientes bajo estatus social son los más adherentes ${ }^{30}$. Se podría decir, que esta variable no está relacionada con la falta de adherencia de los pacientes al tratamiento.

Solo hemos encontrado tres artículos que analizan las creencias en la necesidad de la medicación y el sentimiento de eficacia del tratamiento. Dos estudios encuentran relación de esta variable con la falta de adherencia al tratamiento ${ }^{26,27}$, considerando ambas que aquellos pacientes que creen presentan más adherencia que los pacientes que no creen. Por el contrario, obtuvimos uno que no encontraba relación con la falta de adherencia ${ }^{21}$. El olvido u otras ocupaciones, es una variable que muestra una falta de adherencia no intencional por parte de los pacientes. Cuatro artículos que hemos encontrado que incluyen esta variable en su estudio encuentran relación de esta variable con la falta de adherencia $27,31,38,39$. Esto es debido a que se les olvida tomarse su medicación o no lo hacen a la hora adecuada, porque permanecen ocupados la mayor parte del tiempo. El olvido de la toma de medicación por parte de los pacientes no tiene nada que ver con género, raza, etc.

Hemos encontrado muchos artículos que aborden la variable de la raza. La mayoría no encuentran relación directa de la raza con la falta de adherencia ${ }^{21,25-27,29,30}$. Tan solo tres estudios ${ }^{33,40,41}$ que encuentran relación demuestran que el grupo que menos se adhirió al tratamiento fueron los no caucásicos.

La adhesión al tratamiento se ve influenciada por el costo de éste ${ }^{43}$. El acceso a medicamentos inmunosupresores es también requisito indispensable previo para la adhesión. Un claro ejemplo es el de la Sanidad Privada de Estados Unidos (EE.UU.), donde tan sólo se cubren los inmunosupresores durante los tres años después del trasplante, a partir del cuarto deben de ser abonados por el paciente lo que presenta un mayor impedimento para muchos ${ }^{44}$. La pérdida de cobertura de seguro a partir de los 3 años post-trasplante es preocupante. Un artículo americano ${ }^{42}$ el cual analiza la adherencia de los pacientes americanos a los tres años postrasplante encuentra que hay una gran pérdida de adherencia por 
parte los pacientes a los 3 años debido a las deficiencias económicas que presentan la mayoría de los pacientes para costear el tratamiento inmunosupresor.

Según diversos estudios ${ }^{22,33,38}$, la adherencia al tratamiento inmunosupresor está fuertemente relacionada con la pérdida del injerto como cabía esperar, sin embargo son tres artículos también $n^{24,30,40}$ los que consideran según sus estudios y análisis que ésta variable no está relacionada con la pérdida del injerto o rechazo agudo. Tras finalizar nuestra revisión no hemos podido sacar nada en claro acerca de esta variable.

\section{Conclusiones}

Las variables que se relacionan directa y claramente con la falta de adherencia son la edad, donde hemos encontrado que los pacientes más jóvenes son el grupo menos adherente. La complejidad del tratamiento se correlaciona de forma negativa, a medida que aumenta la complejidad del tratamiento disminuye la adherencia, igual que ocurre con el tiempo postrasplante. El costo del tratamiento es prevalente en la falta de adherencia para aquellas personas que el seguro no les cubre dicho tratamiento.

Por otro lado, en cuanto al estado civil o modo de vida, efectos secundarios, nivel socioeconómico y educativo, situación laboral y raza, no existen resultados concluyentes, ya que la mayoría de los estudios consideran que estas variables no son influyentes en la adherencia al tratamiento y unos contradicen a otros.

En cuanto al género, podemos concluir que es claramente la única variable que no influye sobre la falta de adherencia.

Recibido: 10 Agosto 2013

Revisado: 20 Agosto 2013

Modificado: 12 Septiembre 2013

Aceptado: 20 septiembre 2013

\section{Bibliografía}

1. Laupacis A, Keown P, Pus N, et al. A study of the quality of life and cost-utility of renal transplantation. Kidney Int. 1996; 50(1):235.
2. Loubeau PR, Loubeau JM, Jantzen R. The economics of kidney transplantation versus hemodialysis. Prog Transplant. 2001; 11 (4):291-7.

3. Wolfe RA, Ashby VB, Milford EL, et al. Comparison of mortality in all patients on dialysis, patients on dialysis awaiting transplantation, and recipients of a first cadaveric transplant. N Engl J Med. 1999; 341(23): 1725-1730

4. Moreso F, Hernández D. ¿Ha mejorado la supervivencia del injerto tras el trasplante renal en la era de la moderna inmunosupresión?. Nefrología. 2013;33(1):14-26

5. De Geest $\mathrm{S}$, Borgermans I, Gemoets $\mathrm{H}$ y cols.: Incidence, determinants and consequences of subclinical noncompliance with immunosuppressive therapy in renal transplant recipients. Transplantation. 1995; 59 (3): 340-7.

6. Informe de Diálisis y Trasplante 2012. Sociedad Española de Nefrología. Consultado el 30-102013. Disponible en: http://www.senefro.org/modules/webstructure/files/reer_datos_2012_sen_bilbao_2013.pdf

7. Hariharan $S$, Johnson $C P$, Bresnahan BA, Taranto $S E$, McIntosh MJ, Stablein D. Improved graft survival after renal transplantación in the United States, 1988 to 1996. N Engl J Med. 2000; 342(9): 605-12.

8. Meier-Kriesche HU, Schold JD, Kaplan D. Longterm renal allograft survival: have we made significant progress or it is time to rethink our analytic and therapeutic strategies? Am J Transplant. 2004; 4(8): 1289-95.

9. Butler JA, Roderick P, Mullee M, Mason JC, Peveler RC. Frequency and impact of nonadherence to immunosuppressants after renal transplantation: A systematic review. Transplantation. 2004; 77(5):769-76.

10. Fine RN, Becker $Y$, De Geest $S$, et al. Nonadherence consensus conference summary report. Am J Transplant. 2009; 9(1): 35-41.

11. Denhaerynck $K$, Burkhalter $F$, Schäfer-Keller $P$, Steiger J, Bock A, De Geest S. Clinical consequences of non adherence to immunosuppressive 
medication in kidney transplant patients. Transpl Int. 2009; 22(4): 441-6.

12. Denhaerynck K, Dobbels F, Cleemput I et al. Prevalence, consequences, and determinants of nonadherence in adult renal transplant patients: A literature review. Transpl Int. 2005; 18(10): 1121-1133.

13. Owens ML, Maxwell JG, Goodnight $J$ y cols. Discontinuance of immunosuppression in renal transplantation. Arch surg. 1975; 110(12): 1450-1451.

14. Armstrong SH, Weiner MF. Non compliance with post-transplant immunosuppression. J psychiatry Med. 1982; 11(1): 89-95.

15. Kiley DJ, Lam CS, Pollak R. A study of treatment compílanse following kidney transplantación. Transplantación. 1993; 55(1): 51-56.

16. Morales JM, Varo E, Lázaro P. Immunosuppressant treatment adherence, barriers to adherence and quality of life in renal and liver transplant recipients in Spain. Clin Transplant. 2012: 26(2): 369-376.

17. Cheng CY, Lin BY-J, Chang K-H, Shu K-H, Wu $M-J$. Awareness of Memory Impairment Increases the Adherence to Immunosuppressants in Kidney Transplant Recipients. Transplantation Proceedings. 2012; 44 (3): 746-748.

18. Prendergast MB, Gaston RS. Optimizing Medication Adherence: An Ongoing Opportunity To Improve Outcomes After Kidney Transplantation. Clin J Am Soc Nephrol. 2010; 5(7): 1305-1311.

19. Contreras F, Espinosa JC, Esguerra GA. Calidad de vida, autoeficacia, estrategias de afrontamiento y adhesión al tratamiento en pacientes con Insuficiencia renal crónica sometidos a hemodiálisis. Psicología y salud. 2008; 18 (2): 165-179.

20. Couzi L, Moulin B, Morin MP, et al. Factors Predictive of Medication Nonadherence After Renal Transplantation: A French Observational Study. Transplantation. 2013; 95(2): 326-332.

21. Russell $C L$, Cetingok $M$, Hamburger $K Q$. Et al. Medication adherence in older renal transplant recipients. Clin Nurs Res. 2010; 19(2): 95-112.
22. Pinsky BW, Takemoto SK, Lentine $K L$, Burroughs TE, Schnitzler MA: Transplant outcomes and economic costs associated with patient noncompliance to immunosuppression. Am J Transplant. 2009; 9(11): 2597-2606.

23. Germani $G$, Lazzaro $S$, Gnoato $F$, et al. Nonadherent Behaviors After Solid Organ Transplantation. Transplantation Proceedings. 2011; 43(1): 318323.

24. Cheng $\mathrm{CY}$, Lin BY-J, Chang $\mathrm{K}-\mathrm{H}$, Shu $\mathrm{K}-\mathrm{H}, \mathrm{Wu}$ $\mathrm{M}-\mathrm{J}$. Awareness of Memory Impairment Increases the Adherence to Immunosuppressants in Kidney Transplant Recipients. Transplantation Proceedings. 2012; 44 (3): 746-748.

25. Cukor D, Rosenthal DS, Jindal RM, Brown CD, Kimmel PL. Depression is an important contributor to low medication adherence in hemodialyzed patients and transplant recipients. Kidney Int. 2009; 75(11):1223-1229.

26. Clifford S, Barber N, Horne R. Understanding different Belief held by adherers, unintentional nonadherers, and intentional nonadherers: Application of the Necessity-Concerns Framework. J Psychosom Res. 2008; 64(1): 1-46.

27. Chisholm-Burns M, Pinsky B, Parker G, et al. Factors related to immunosuppressant medication adherence in renal transplant recipients. Clin Transplant.. 2012; 26(5): 706-713.

28. Gremigni $P$, Bacchi $F$, Turrini $C$. et al. Psychological factors associated with medication adherence following renal transplantation. Clin Transplant. 2007; 21 (6): 710-715.

29. Russell CL, Conn VS, Ashbaugh C. Et al. Medication Adherence Patterns in Adult Renal Transplant Recipients. Research in Nursing \& Health. 2006; 29(6): 521-532.

30. Chisholm MA, Lance CE, Mulloy LL. Patient factors associated with adherence to immune suppressant therapy in renal transplant recipients. Am J Health-Syst Pharm. 2005; 62(17): 1775-1781.

31. Griva K, Davenport A, Harrison M, Newman SP. Non-adherence to Immunosuppressive Medications in Kidney Transplantation: Intent Vs. Forgetfulness 
and Clinical Markers of Medication Intake. ann. behav. med. 2012; 44(1): 85-93.

32. Dharancy S, Giral M, Tetaz R, et al. Adherence with immunosuppressive treatment after transplantation: results from the French trial PREDICT. Clin Transplant. 2012; 26 (3): 293-299.

33. Nevins TE. Thomas W. Quantitative Patterns of Azathioprine Adherence After Renal Transplantation. Transplantation. 2009; 87(5): 711-718.

34. M.M.T. Brahm, R.C. Manfro, D. Mello, S. Cioato, and L.F.S. Gonçalves. Evaluation of Adherence to Immunosuppressive Drugs in Kidney Transplantation by Control of Medication Dispensing. Transplantation Proceedings. 2012; 44(8): 2391-2393.

35. Chisholm-Burns MA, Kwong WJ, Mulloy LL, Spivey CA. Nonmodifiable characteristics associated with nonadherence to immunosupresante therapy in renal transplant recipients. Am J Health Syst Pharm 2008; 65(13): 1242-1247.

36. Chisholm MA, Williamson GM, Lance CE, Mulloy LL. Predicting adherence to immunosuppressant therapy: A prospective analysis of the theory of planned behaviour. Nephrol Dial Transplant. 2007; 22(8): 2339-2348.

37. N. Ichimaru, Y. Kakuta, T. Abe. Et al. Treatment Adherence in Renal Transplant Recipients: A Questionnaire Survey on Immunosuppressants. Transplantation Proceedings. 2008; 40 (5): 1362-1365.

38. Butler JA, Roderick P, Mullee M, Mason JC, Peveler $\mathrm{RC}$. Frequency and impact of nonadherence to immu- nosuppressants after renal transplantation: A systematic review. Transplantation 2004; 77(5): 769-76.

39. Schmid-Mohler G, Thut MP, Wüthrich RP, Denhaerynck K, De Geest S. Non-adherence to immunosuppressive medication in renal transplant recipients within the scope of the integrative model of behavioral prediction: a cross-sectional study. Clin Transplant. 2010; 24 (2): 213-222.

40. Israni $A K$, Weng $F L$, Cen $Y-Y$, Joffe $M$, Kamoun $M$, Feldman HI. Electronically measured adherence to immunosuppressive medications and kidney function after deceased donor kidney transplantation. Clin Transplant. 2011: 25(2): 124-131.

41. Weng $F L$, Israni $A K$, Joffe $M M$, Hoy $T$, Gaughan CA. Race and electronically measured adherence to immunosuppressive medications after deceased donor renal transplantation. J Am Soc Nephrol. 2005; 16(6): 1839-1848.

42. Evans $R$, Applegate $W$, Briscoe $D$, et al. Cost-Related Immunosuppressive Medication Nonadherence Among Kidney Transplant Recipients. Clin J Am Soc Nephrol. 2010. 5(12): 2323-2328.

43. Hilbrands LB, Hoitsma AJ, Koene R: Medication compílanse after renal transplantation. Transplantation. 1995; 60 (9): 914-920.

44. De Geest $\mathrm{S}$, Borgermans I, Gemoets $\mathrm{H}$ et al. Incidence, determinants and consequences of subclinical noncompliance with immunosuppressive therapy in renal transplant recipients. Transplantation. 1995; 59 (3): 340-347. 